\title{
Successful Removal of a Metastatic Gastrointestinal Stromal Tumor in the Craniovertebral Junction using an Occipital Artery to Posterior Inferior Cerebellar Artery Bypass
}

\author{
Yukitomo Ishi Naoki Nakayama Hiroyuki Kobayashi \\ Shigeru Yamaguchi Shunsuke Terasaka Kiyohiro Houkin \\ Department of Neurosurgery, Graduate School of Medicine, Hokkaido University, \\ Sapporo, Japan
}

\section{Key Words}

Craniovertebral junction - Gastrointestinal stromal tumor - Metastasis - Occipital artery to posterior inferior cerebellar artery bypass . Prophylactic vascular reconstruction - Tumor resection

\begin{abstract}
Gastrointestinal stromal tumors (GISTs) are rare mesenchymal neoplasms arising from the gastrointestinal tract. The authors present a case of the successful removal of a metastatic GIST in the craniovertebral junction, using an occipital artery to posterior inferior cerebellar artery (OA-PICA) bypass. The patient is a 54-year-old male who underwent his first surgery for a small-bowel tumor at the age of 45 and was diagnosed with GIST. Nine years after his primary diagnosis, the patient suffered from severe neck pain. MRI demonstrated a large demarcated mass adjacent to the right atlas. The right vertebral artery (VA), completely engulfed by the tumor, showed a narrowing and ended in the PICA. Poor collateral blood supply in the right PICA territory was presumed. To prevent ischemic complications, an OAPICA bypass was performed prior to the tumor resection. After the OA-PICA bypass, the tumor associated with the right VA was successfully removed, and the patient was discharged without any neurological deficits.


Ishi et al.: Removal of a Metastatic GIST Using OA-PICA Bypass

\section{Introduction}

Gastrointestinal stromal tumors (GISTs) are a rare mesenchymal neoplasm arising from the gastrointestinal tract. Distant metastasis is not rare, but it is usually found in the liver or peritoneum. Metastasis of the head and neck region is relatively rare, and only a few scattered reports are available on the subject [1-7]. GISTs were previously known to be refractory to conventional chemotherapy and radiotherapy; however, the discovery of gainof-function mutations in KIT proto-oncogene dramatically revealed the molecular pathogenesis of this tumor and identified KIT as a possible suitable target for molecular target therapy [8-10]. Although several tyrosine kinase inhibitors (TKIs) for the treatment of GISTs are available, surgical resection still has a major role to play in local disease control. We present a case with radical surgical resection of a metastasized lesion, using an OA-PICA bypass.

\section{Case Report}

A 54-year-old male first underwent surgery for a small-bowel tumor at the age of 45 and was diagnosed with GIST. Two years later, the tumor was recurrent as a metastatic liver tumor. Although the patient had to go through a resection of his metastatic liver and peritoneal tumors of GIST several times, he was able to maintain a stable status in his performance. Chemotherapy with TKIs, such as imatinib mesylate (Gleevec/Glivec ${ }^{\circledR}$ ) and sunitinib malate (Sutent ${ }^{\circledR}$ ), was frequently interrupted by their severe side effects.

Nine years after his primary diagnosis, the patient suffered from severe neck pain. MRI of the cervical spine showed an abnormal mass in the craniovertebral junction, and he was referred to our department. There was no neurological deficit except for his neck pain. MRI demonstrated a large demarcated mass with a maximum diameter of $45 \mathrm{~mm}$ adjacent to the right atlas (fig. 1a, b). The tumor appeared to be hypointense on T1-weighted and hyperintense on T2-weighted images. The mass was slightly enhanced with contrast material. A CT revealed the mass engulfing the right transverse process of the atlas and a narrowed right vertebral artery (VA) (fig. 1c). 3D-CT angiography revealed that the right VA ended in PICA and the distal segment of the right VA showed hypoplasia (fig. 1d). Since GISTs are known to be resistant to irradiation [9], we decided to perform an extensive tumor resection to prolong his survival. Avoiding ischemic complications, we planned a prophylactic ipsilateral OA-PICA bypass prior to tumor resection. This vascular reconstruction would enable us not only to prevent cerebellar infarction, but also to perform an en-bloc resection together with the right VA, which was presumably invaded by the tumor.

The patient was placed in a lateral position, and a right high cervical dissection was performed. After we accomplished the OA-PICA anastomosis, the tumor, located under the posterior cervical muscles, was exposed. The tumor was soft and its surface was smooth. As we had assumed, the boundary between the tumor and the surrounding tissue was clear, whereas the right transverse process of the atlas and the VA were completely engulfed by the tumor. The tumor associated with the right VA was completely removed in an en-bloc fashion.

Pathological examination showed spindle-shaped tumor cells exhibiting a bundle-like form (fig. 2a). In immunohistochemistry, these tumor cells showed a high expression of KIT (fig. 2b), indicating that the tumor was consistent with the metastasis of GIST. Post-operative MRI presented no evidence of cerebellar infarction or a tumor remnant, and the good patency of the OA graft was confirmed by 3D-CT angiography. The patient was discharged 
Ishi et al.: Removal of a Metastatic GIST Using OA-PICA Bypass

with no neurological deficits. The other TKIs, sorafenib tosylate (Nexavar ${ }^{\circledR}$ ) and regorafenib hydrate, were both applied as third- and fourth-line chemotherapy. Postoperative radiotherapy was not performed. The patient is working as of 22 months post-surgery.

\section{Discussion}

GISTs are rare mesenchymal tumors that account for less than $1 \%$ of all gastrointestinal tumors, but they are the most common of the gastrointestinal mesenchymal tumors. The major sites of primary origin are the stomach (39\%) and the small intestine (32\%). Distant metastasis of GISTs occurs in about 50\% of the cases, but it is usually found in the liver and the peritoneum $[1,11]$. Due to drug resistance, although TKIs as molecular target agents have improved the prognosis of this rare entity, metastatic or recurrent GISTs still are difficult to treat effectively, and radical resection may be the only feasible treatment option.

Regardless of its primary origin or metastatic condition, cranial base tumors sometimes encase the internal carotid artery or the VA, where they invade the vessel wall. Therefore, an invasive procedure might put the patient at risk for vascular injury. Cerebral revascularization in cases with cranial base tumors is sometimes performed, but cerebellum revascularization is even less common [12,13]. Although there has been controversy over prophylactic vascular reconstruction in brain tumor surgeries, our strategy for performing this procedure is relatively straightforward. In cases with radiological findings, the criteria are as follows: first, major vessels are engulfed by the tumor and show a narrowing or an elongation in the angiogram; second, poor collateral blood supply is presumed when the affected vessel is interrupted, and third, if the tumor is histologically malignant, we perform bypass surgery prior to tumor resection, regardless of whether or not the circulation is anterior or posterior. A deliberate surgical strategy should be outlined, especially for patients with malignancy, because they do not have enough time for postoperative rehabilitation. Our case met the above criteria, and we selected an OA-PICA bypass for the cerebellum revascularization. Collaboration of skull base surgery and neurovascular surgery greatly contributed to the surgical outcome of this patient. A metastasized GIST in the craniovertebral junction was successfully removed without any neurological or radiological deficits.

\section{Conclusion}

We reported a case of GIST metastasized in the craniovertebral junction. The right VA was engulfed by the tumor, and the OA-PICA bypass prior to tumor removal was significantly effective to avoid postoperative ischemic complications.

\section{References}

1 Baeg MK, Bae SH, Lee KH, Kim J, Park IS, Jin JY: Diplopia as a presenting symptom in a gastric gastrointestinal stromal tumor. Jpn J Clin Oncol 2011;41:265-268.

-2 Gil-Arnaiz I, Martinez-Trufero J, Pazo-Cid RA, Felipo F, Lecumberri MJ, Calderero V: Skull metastasis from rectal gastrointestinal stromal tumours. Clin Transl Oncol 2009;11:625-627.

3 Hamada S, Itami A, Watanabe G, Nakayama S, Tanaka E, Hojo M, et al: Intracranial metastasis from an esophageal gastrointestinal stromal tumor. Intern Med 2010;49:781-785.

4 Hughes B, Yip D, Goldstein D, Waring P, Beshay V, Chong G: Cerebral relapse of metastatic gastrointestinal stromal tumor during treatment with imatinib mesylate: case report. BMC Cancer 2004; 4:74.

$\checkmark 5$ Kaku S, Tanaka T, Ohtuka T, Seki K, Sawauchi S, Numoto RT, et al: Perisacral gastrointestinal stromal tumor with intracranial metastasis. Case report. Neurol Med Chir (Tokyo) 2006;46:254-257. 


\begin{tabular}{l|l}
\hline \multicolumn{2}{l}{ Case Rep Neurol 2014;6:139-143 } \\
\hline DOI: 10.1159/000362867 & $\begin{array}{l}\text { C 2014 S. Karger AG, Basel } \\
\text { www.karger.com/crn }\end{array}$ \\
\hline
\end{tabular}

Ishi et al.: Removal of a Metastatic GIST Using OA-PICA Bypass

-6 Naoe H, Kaku E, Ido Y, Gushima R, Maki Y, Saito H, et al: Brain metastasis from gastrointestinal stromal tumor: a case report and review of the literature. Case Rep Gastroenterol 2011;5:583-589.

7 Puri T, Gunabushanam G, Malik M, Goyal S, Das AK, Julka PK, et al: Mesenteric gastrointestinal stromal tumour presenting as intracranial space occupying lesion. World J Surg Oncol 2006;4:78.

8 Hirota S, Isozaki K, Moriyama Y, Hashimoto K, Nishida T, Ishiguro S, et al: Gain-of-function mutations of c-kit in human gastrointestinal stromal tumors. Science 1998;279:577-580.

9 Rammohan A, Sathyanesan J, Rajendran K, Pitchaimuthu A, Perumal SK, Srinivasan U, et al: A gist of gastrointestinal stromal tumors: a review. World J Gastrointest Oncol 2013;5:102-112.

10 Willmore C, Holden JA, Zhou L, Tripp S, Wittwer CT, Layfield LJ: Detection of c-kit-activating mutations in gastrointestinal stromal tumors by high-resolution amplicon melting analysis. Am J Clin Pathol 2004;122:206-216.

11 DeMatteo RP, Lewis JJ, Leung D, Mudan SS, Woodruff JM, Brennan MF: Two hundred gastrointestinal stromal tumors: recurrence patterns and prognostic factors for survival. Ann Surg 2000;231:51-58.

12 Ramanathan D, Temkin N, Kim LJ, Ghodke B, Sekhar LN: Cerebral bypasses for complex aneurysms and tumors: long-term results and graft management strategies. Neurosurgery 2012;70:1442-1457.

-13 Sekhar LN, Natarajan SK, Ellenbogen RG, Ghodke B: Cerebral revascularization for ischemia, aneurysms, and cranial base tumors. Neurosurgery 2008;62:1373-1408.
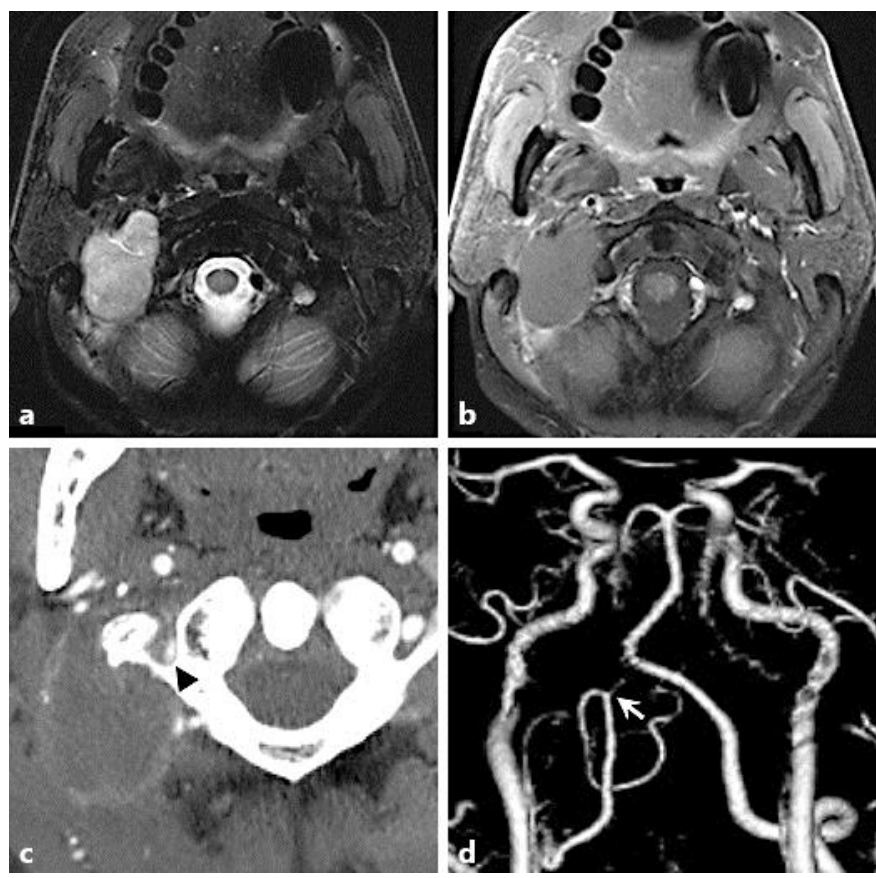

Fig. 1. MRI demonstrating a tumor located in the right craniovertebral junction. a Axial T2-weighted image. b Axial gadolinium-enhanced fat-suppressed T1-weighted image. c Contrast-enhanced CT demonstrates the right transverse process of the atlas surrounded by the tumor and narrowed VA at the right transverse foramen (black arrowhead). d 3D-CT angiography showing the right VA ended in PICA (arrow). 
Ishi et al.: Removal of a Metastatic GIST Using OA-PICA Bypass

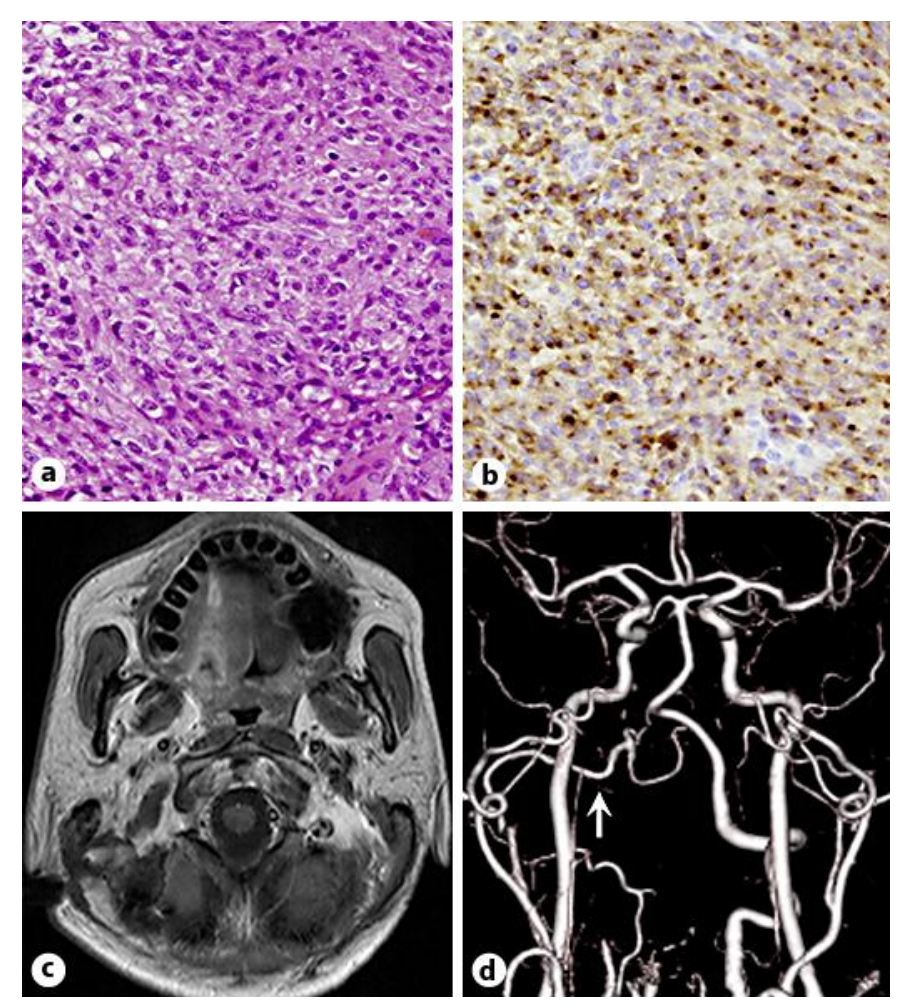

Fig. 2. a Histopathology with HE staining of the tumor shows spindle-shaped tumor cells exhibiting a bundle-like form. b Immunohistochemical staining demonstrates the KIT expression in tumor cells. c Postoperative gadolinium enhanced T1-weighted image presenting no evidence of residual tumors. $\mathbf{d}$ Postoperative 3D-CT angiography showing a patency of occipital artery graft (arrow). 